\title{
THE WR/WRprogenitor NUMBER RATIO: THEORY AND OBSERVATIONS.
}

\author{
D. VANBEVEREN \\ Dept. of Physics, VUB, \\ Brussels, Belgium.
}

SUMMARY. A direct comparison between the observed WR/WRprogenitor number ratio within $2.5 \mathrm{kpc}$ from the sun and the predicted value (using evolutionary computations of single stars of Maeder and Meynet, 1987, A.\&A. 182, 243) reveals a discrepancy of at least a factor of two. In a previous study (Vanbeveren, 1990, A.\&A. in press) I proposed a solution based on the incompleteness of the observed OB type star sample within $2.5 \mathrm{kpc}$ from the sun. In this summary, I propose a theoretical explanation for the discrepancy. The theoretically predicted WR/WR progenitor number ratio critically depends on the adopted $\dot{M}$ formalism in evolutionary computations during the red supergiant phase (RSG) of a massive star, especially in the mass range $20-40 \mathrm{M}_{\boldsymbol{\Theta}}$. Since any $\dot{M}$ formalism predicts the mass loss rate with an uncertainty of at least a factor of two, I have tried to look for solutions for the WR/WR progenitor problem by using different values of $\dot{M}$ during the RSG (in the mass range $20-40 \mathrm{M}_{\Theta}$ ); the $\dot{M}$ values and formalism that were adopted were always choosen within the observational uncertainty (i.e. within a factor of two when compared to the formalism used by Maeder and Meynet, 1987).

\section{The results.}

When we allow for a $\sim 40 \%$ increase of the RSG $\dot{M}$ values in the mass range $20-40 \mathrm{M}_{\odot}$ when compared to the values used by Maeder and Meynet (1987), it can be concluded that

a. independent from whether a WR star is a single star or whether it is a member of a close binary, theoretical evolution predicts that WR stars originate from stars with initial ZAMS mass larger than $20 \mathrm{M}_{\odot}$ although the bulk descends from the $\mathbf{M}>25-30 \mathrm{M}_{\odot}$ range corresponding to observations.

b.the theoretically predicted WR/WR progenitor number ratio corresponds to the observed value within $2.5 \mathrm{kpc}$ from the sun.

General conclusion: accounting for the uncertainty of the stellar wind mass loss rate in massive red supergiants and for the possible incompleteness of the $O B$ type star sample within $2.5 \mathrm{kpc}$ from the sun, the theoretically predicted $\mathrm{WR} / \mathrm{WR}$ progenitor number ratio corresponds to the observed value. 